\title{
SINGULARITIES OF PIECEWISE LINEAR MAPPINGS. I MAPPINGS INTO THE REAL LINE
}

\author{
BY A. KOSINSKI ${ }^{1}$ \\ Communicated by Edwin Spanier, November 27, 1961
}

In this note we consider piecewise linear mappings of combinatorial manifolds into the real line. We define - in a purely combinatorial way - a certain class of mappings called nondegenerate and we prove that every continuous mapping may be approximated by a nondegenerate one. Nondegenerate mappings on a combinatorial manifold behave like differentiable nondegenerate mappings on a differentiable manifold. In particular, the index of a singularity can be defined and one can prove the Morse inequalities and an analogue of the Reeb theorem about a function with only two nondegenerate singularities.

The approximation theorem can also be extended to height functions on combinatorial submanifolds of Euclidean space.

Detailed proofs will be published later. We give here descriptions of the singularities, a statement of the main theorems and a very brief sketch of the approximation theorem.

1. $E^{n}$ will denote $n$-dimensional Euclidean space. By a complex we shall understand a rectilinear, locally finite simplicial complex in Euclidean space. A closed subspace $M \subset E^{n}$ will be said to be a manifold if $M$ is a space of a complex $K$ which is a connected combinatorial manifold in the sense of M.H.A. Newman and J. W. Alexander (see [1]). We will then say that $K$ is a triangulation of $M$. The boundary $\dot{M}$ of $M$ will be the subset of $M$ covered by the mod 2 boundary of its triangulation; if $\dot{M}=\varnothing$ we will say that $M$ is unbounded, a compact and unbounded manifold will be said to be closed.

Let $U, V$ be open subsets of a manifold $M$ and let $f: V \rightarrow M_{1}$ be a continuous mapping of $V$ into a manifold $M_{1}$. We say that $f$ is piecewise linear (shortly: PL) in $U$ if there exist triangulations $K$ of $M$ and $K_{1}$ of $M_{1}$ such that for every simplex $\Delta$ of $K$ intersecting $U$, $f$ maps $\triangle \cap V$ linearly into a simplex of $K_{1}$. If $U=V=M$ we will say that $f$ is a PL mapping of $M$. If $p \in V \subset M$ and $f: V \rightarrow M_{1}$ is PL in a certain neighborhood of $p$ then we will say that $f$ is $\mathrm{PL}$ at $p$.

Let $f_{i}: M_{i} \rightarrow E^{k}$ be two PL-mappings, and let $p_{i} \in M_{i}, i=1,2$. We say that $f_{1}$ at $p_{1}$ is equivalent to $f_{2}$ at $p_{2}$ if there exist neighborhoods $U_{i}$ of $p_{i}, i=1,2$ and homeomorphisms $h: E^{k} \rightarrow E^{k}, g: U_{1} \rightarrow U_{2}$ such that

1 This work was supported by the National Science Foundation research grant NSF G-18919. 
(a) $g$ is PL at $p_{1}, g\left(p_{1}\right)=p_{2}$;

(b) $h$ is $\mathrm{PL}$ at $f_{2}\left(p_{2}\right), h$ is orientation preserving;

(c) $h f_{1}(x)=f_{2} g(x)$ for $x \in U_{1}$.

We write then $f_{1}\left|p \approx f_{2}\right| q$.

By a special $n$-cell $Q^{n}$ we will understand the convex hull of the set of points $a_{i}=\left(a_{i 1}, \cdots, a_{i n}\right) \in E^{n}, i=1, \cdots, 2 n$, where $a_{i j}=\delta_{i}^{j}$ for $i=1, \cdots, n, j=1, \cdots, n$ and $a_{i j}=-\delta_{i-n}^{j}$ for $i=n+1, \cdots, 2 n$, $j=1, \cdots, n$. By a special $(n-1)$-sphere $S^{n-1}$ we will understand the boundary of $Q^{n}$. It is easy to see that the points $a_{i}, i=1, \cdots, 2 n$ triangulate $S^{n-1}$ and that the same set of points with the addition of $a_{0}=(0, \cdots, 0)$ triangulates $Q^{n}$. We will refer to these triangulations as the standard ones.

By $\alpha: E^{n} \rightarrow E^{n}$ we denote the symmetry of $E^{n}$ with respect to the origin.

We consider the class $C$ of those mappings of $Q^{n}$ into the real line which map $a_{0}$ into $0, a_{i}$ into $\pm 1, i=1, \cdots, 2 n$, and are linear on every simplex of the standard triangulation.

A mapping $f \in C$ will be said to be symmetric if $f \alpha\left(a_{i}\right)=f\left(a_{i}\right)$, $i=1, \cdots, 2 n$. Let $C_{k}, k=0, \cdots, n$, be the class of symmetric maps which map exactly $2 k$ vertices into -1 , let $C_{n+1}$ be the class of nonsymmetric maps.

The following lemmas explain the structure of maps in $C$ :

Lemma 1.1. Let $f \in C_{k}, g \in C_{l}$. Then $f\left|a_{0} \approx g\right| a_{0}$ iff $k=l$.

Lemma 1.2. Let $p \in Q^{n}-S^{n-1}, p \neq a_{0}$ and let $f \in C$. Then $f|p \approx g| a_{0}$ where $g$ is an arbitrary mapping from $C_{n+1}$.

Now, we can define nondegenerate mappings and singularities.

Definition. Let $f: M \rightarrow E^{1}$ be a PL mapping of a manifold $M$ into the line. Let $p \in M$. We say that $f$ is regular at $p$ if $f\left|p \approx f_{n+1}\right| a_{0}$, $f_{n+1} \in C_{n+1}$. We will say that $f$ has a nondegenerate singularity of index $k$ at $p$ if for some $k \leqq n, f\left|p \approx f_{k}\right| a_{0}, f_{k} \in C_{k}$. We will say that $f$ is nondegenerate if $f$ has nondegenerate singularities at isolated points and is regular elsewhere.

In [5] M. Morse defined $T$-critical and $T$-ordinary points of a continuous mapping of a topological manifold into the real line. Comparison of his theory with the above definition gives

Lemma 1.3. Let $f: M \rightarrow E^{1}$ be a PL mapping of a manifold into the real line, let $p \in M$. If $f$ is regular at $p$ then $p$ is a T-ordinary point of $f$; if $f$ has a nondegenerate singularity of index $k$ at $p$, then $p$ is a T-critical point of $f$ of index $k$ in the sense of $M$. Morse. 
2. In the last section we give a brief sketch of the proof of the following approximation theorem:

THEOREM 2.1. Every continuous mapping of an unbounded manifold into the line may be approximated arbitrarily closely by a nondegenerate mapping.

A special class of mappings of manifolds into the line is given by so-called height functions. If $M$ is a manifold in a Euclidean space $E^{n}$ and if $L$ is a line in $E^{n}$ then the height function $h_{L}$ on $M$ is the orthogonal projection of $M$ into $L$.

Theorem 2.2. Let $f: M \rightarrow E^{n}$ be a PL homeomorphism of a closed manifold and let $L$ be a line in $E^{n}$. Then arbitrarily near to $f$ there exists $a \mathrm{PL}$ homeomorphism $\mathrm{g}: M \rightarrow E^{n}$ such that the height function $h_{L}$ is nondegenerate on $g(M)$.

Dr. Gluck informed me that he had proved a similar theorem in the case of a 2-manifold in $E^{4}$ (see [2]).

Lemma 1.3 permits application of results of M. Morse [5] to nondegenerate mappings. For instance:

Theorem 2.3. Morse inequalities are valid for nondegenerate functions on closed manifolds.

For the definition of Morse inequalities see e.g. [4].

TheOREM 2.4. Let $M$ be a closed n-dimensional manifold and $f$ a nondegenerate mapping of $M$ into the line with exactly two singular points. Then there exists a PL homeomorphism of $M$ onto $S^{n}$.

G. Reeb proved the corresponding theorem for the differentiable case (see e.g. [4]), and N. H. Kuiper for the topological case [3]. It follows from the theorem of Kuiper together with Lemma 1.3 that $M$ is homeomorphic to $S^{n-1}$. However, in the proof of Theorem 2.4 we do not use this result but instead apply the following

LeMma 2.5. Let $f: M \rightarrow E^{1}$ be a nondegenerate mapping and $c_{1}, c_{2}$, $c_{1} \neq c_{2}$, two points in $E^{1}$ such that has no critical points in $A=f^{-1}\left(\left[c_{1}, c_{2}\right]\right)$. Then $A$ is a regular neighborhood (in the sense of $J . H$. C. Whitehead [6]) of $f^{-1}\left(c_{1}\right)$.

3. Henceforth, triangulation will mean Brouwer triangulation (see [7]). If $K$ is a triangulation of a manifold $M$ and $f: M \rightarrow E^{k}$ a mapping which is linear on every simplex of $K$, we will say that $f$ is semi-linear on $K$. 
The proof of Theorem 2.1 starts with the following

LEMMA 3.1. If $f: M \rightarrow E^{1}$ is semilinear on a triangulation $K$ and if $f(p) \neq f(q)$ for every two distinct vertices $p, q$ of $K$, then $f$ has only isolated singular points.

It is easy to obtain an approximation to a given mapping $f: M \rightarrow E^{\mathbf{1}}$ which satisfies the condition of 3.1. Therefore the proof of 2.1 reduces to the following "local" lemma:

LEMMA 3.2. Let $M$ be a combinatorial cell and $K$ a star triangulation of $M$, i.e. $K=a \dot{K}$. Let $f: M \rightarrow E^{1}$ be a semilinear mapping with only one singularity at $a$. Then there exists an arbitrarily close approximation to $f$ which has only nondegenerate singularities and agrees with $f$ on a certain neighborhood of $\dot{M}$.

The proof of 3.2 is by induction on the dimension of $M$. We first find in $M$ two "concentric" cells $M_{1}, M_{2}$ such that $M_{1} \subset M_{2}-\dot{M}_{2}$, $M_{1}$ is small and has the property that $f \mid \dot{M}_{1}$ has only isolated singularities. Those singularities will appear only at points $q_{i}$ which are intersections of segments $\left[a p_{i}\right]$ with $\dot{M}_{1}$, where $p_{i}$ is a vertex of $\dot{K}$.

Now, let $\dot{K}_{1}^{\prime}$ be the first barycentric subdivision of $\dot{K}_{1}$, let $S_{i}$ be the star of $q_{i}$ in $\dot{K}_{1}^{\prime}$ and let $S_{i}^{0}$ be the 0 -sphere consisting of points $a$, $t_{i}$, where $t_{i}=\dot{M}_{2} \cap\left[a p_{i}\right]$. The complex $S_{i} S_{i}^{0}$ is then a triangulation of a certain (closed) neighborhood $N_{i}$ of $q_{i}$ in $M_{2}$. Now, by the inductive assumption we can approximate $f \mid S_{i}$ by a mapping $g_{i}$ semilinear on a subdivision $S_{i}^{\prime}$ of $S_{i}$ with nondegenerate singularities only and agreeing with $f$ on a neighborhood of $\dot{S}_{i}$ in $S_{i}$. The mappings $g_{i}$ define together a mapping $g: \dot{M}_{1} \rightarrow E^{1}$. Let $\alpha_{1}>\alpha=\max _{x \in \dot{M}_{1}} g(x)$, we suppose $\alpha_{1}-\alpha$ to be small. The mapping $h: M \rightarrow E^{1}$ is defined by the following conditions:

(a) $h(a)=\alpha_{1}, h\left(t_{i}\right)=f\left(t_{i}\right), h \mid \dot{M}_{1}=g$;

(b) $h \mid N_{i}$ is semilinear on $S_{i}^{\prime} S_{0}$;

(c) $h(x)=f(x)$ for $x \in M-U N_{i}$.

The mapping $h$ is then the desired approximation to $f$.

Let us remark finally that it is obvious that nondegenerate mappings do not form an open set in the space of all PL-mappings of a given compact manifold $M$. However, we have a certain kind of stability with respect to a given triangulation. Namely:

TheOREM 3.3. Let $f: M \rightarrow E^{1}$ be nondegenerate and semi-linear on a triangulation $K$ of $M$. Let $K^{\prime}$ be a subdivision of $K$. There exists an $\epsilon>0$ such that if $g: M \rightarrow E^{1}$ is semilinear on $K^{\prime}$ and $\rho(g, f)<\epsilon$ then for every $p \in M, f|p \approx g| p$. 


\section{REFERENCES}

1. J. W. Alexander, The combinatorial theory of complexes, Ann. of Math. 31 (1930), 292.

2. H. Gluck, The embedding of two-spheres in the four-sphere, to appear.

3. N. H. Kuiper, A continuous function with two critical points, Bull. Amer. Math. Soc. 67 (1961), 281.

4. J. Milnor, Morse theory, Lecture notes, Princeton Univ., 1960.

5. M. Morse, Topologically non-degenerate functions on a compact $n$-manifold, J. Analyse Math. 7 (1959), 189.

6. J. H. C. Whitehead, Simplicial spaces, nuclei and m-groups, Proc. London Math. Soc. 45 (1939), 243.

7. - Note on manifolds, Quart. J. Math. Oxford Ser. (2) 12 (1941), 26.

University of California, Berkeley 